\title{
Convexity Adjustments for ATS models *
}

\author{
Raquel M. Gaspar \\ Advance Research Center, \\ ISEG, Technical University Lisbon, \\ Rua Miguel Lupi 20, room 101, \\ 1249 Lisbon, \\ PORTUGAL \\ Rmgaspar@iseg.utl.pt
}

\author{
Agatha Murgoci \\ Department of Finance, \\ Stockholm School of Economics, \\ P.O.Box 6501, \\ SE-113 83 Stockholm, \\ SWEDEN \\ agatha.murgoci@hhs.se
}

ADVANCE working paper Series, n. 9/2008

\begin{abstract}
Practitioners are used to value a broad class of exotic interest rate derivatives simply by adjusting for what is known as convexity adjustments (or convexity corrections). We start by exploiting the relations between various interest rate models and their connections to measure changes. As a result we classify convexity adjustments into forward adjustments and swaps adjustments.

We, then, focus on affine term structure (ATS) models and, in this context, conjecture convexity adjustments should be related of affine functionals. In the case of forward adjustments, we show how to obtain exact formulas. Concretely for LIBOR in arrears (LIA) contracts, we derive the system of Riccatti ODE-s one needs to compute to obtain the exact adjustment. Based upon the ideas of Schrager and Pelsser (2006) we are also able to derive general swap adjustments useful, in particular, when dealing with constant maturity swaps (CMS).

Our approach bypasses the need for Taylor approximations or unrealistic assumptions. They include exact convexity adjustments previously derived, such as the adjustments associated with Gaussian models, but are far more general as they provide solutions for the entire ATS class of models.
\end{abstract}

JEL Classification: C02, C65, E43, G12

Key words: affine term structure, convexity adjustments, CMS, LIBOR in arrears

\footnotetext{
${ }^{*}$ We are particularly indebted to Rama Cont for showing us how interesting convexity adjustments may be and for his insights on the subject. We thank Tomas Björk for helpful comments.

The financial support of FCT under grant PTDC/MAT/64838/2006 and Jan Wallander's Foundation is gratefully acknowledged.
} 


\section{Contents}

1 Introduction $\quad 3$

2 Convexity adjustment connection to measure changes 4

3 Linking different Interest Rate Models 5

3.1 Forward martingale measures, HJM and LIBOR market models . . . . . . . . . . 6

3.2 Swap martingale measures and swap market models . . . . . . . . . . . . . . . 7

4 Convexity Adjustments for ATS models 9

4.1 Forward Convexity Adjustments . . . . . . . . . . . . . . . . . . . . . . . 10

4.1 .1 Expectation Hypothesis adjustment ． . . . . . . . . . . . . 10

4.1 .2 LIA adjustment . . . . . . . . . . . . . . . . . . . 12

4.2 Swap Convexity Adjustments . . . . . . . . . . . . . . . . . . . . . . . . 14

4.2 .1 CMS adjustment . . . . . . . . . . . . . . . . . . . 15

\section{Notation}

- $r_{t}$ : instantaneous short rate of interest

- $f(t, T)$ : instantaneous forward rate of interest

- $f(t, T, S)$ : continuously compounding forward rate for the period $(T, S)$

- $L(t, T, S)$ : simply compounded forward (LIBOR) rate at time $t$ for the period $(T, S)$

- $L(t, T)=L(t, t, T)$ : simply compounding spot rate over the period $(t, T)$

- $r(t, T)$ : continuously compounding spot rate over the period $(t, T)$

- $S\left(t, T_{0}, T_{N}\right)$ : forward swap rate contracted at time $t$ with an effective period starting at $T_{0}$ and involving periodic payments at $\left.T_{1}, \cdots, T_{N}\right)$

- $\alpha_{i}$ : Time gap between two tenors $\alpha_{i}=T_{i-1}-T_{i}$

- $S(t, T)=S(t, t, T)$ : spot swap rate at time $t$ of a swap with first reset date $t$ and payment dates $t+1 \cdots T$

- $p(t, T)$ : price, at time $t$, of a zero coupon bond that pays 1 at $T$

- $P$ : a portfolio of several zero-coupon bonds with different maturities. 


\section{Introduction}

The notion of convexity adjustment in fixed income markets arises when one uses prices of standard (plain vanilla) products corrected by an adjustment to price nonstandard products. Despite their non-standard features, these products are many times quite similar to plain vanilla ones whose price can either be directly obtained from the market or at least computed in closedform. Thus, adjustments of plain vanilla prices can be understood as "corrections" needed to capture the bias introduced in prices by the complexities of the non-standard products.

From a theoretical point of view, the appropriate way to look into convexity adjustments is as a consequence of a measure change, as proposed by Plesser (2003). Due to the non-standard features in fixed income products,computing prices is equivalent to computing expectations under the "wrong" measure. This leads to difficulties in obtaining closed-form solutions. The industry practice is then to first compute the price as if our payoff would have "nice" martingale properties and then approximate the necessary convexity adjustments using Taylor approximations. Examples of such adjustment can be found in approximations for pricing formulae of products such as in-arrears or in-advance products, constant maturity swaps (CMS) products, or equity swaps. Popular approximations are the ones proposed in Hull (2006) or by Pugachevsky (2001), Hart (1997), Hagan (2003), Pelsser (2000) and Brigo and Mercurio (2006).

Very little have been theoretically derived in closed form, except for the case of Gaussian interest rates. For studies on Gaussian interest rate models we refer to Jamshidian (1997), Kennedy (1997) , Pugachevsky (2001) or Hunt and Kennedy (2000), for instance.

The purpose of this study is to go one step further on the derivation of exact convexity adjustments exploiting their connections with measure changes. For the entire class of affine term structure (ATS) models we are able to derive exact results for popular convexity adjustments, including LIBOR in-arrears (LIA) and CMS adjustments.

The remaining of this paper is organized as follows.

In Section 2, we introduce the basic notation and formalize the link between convexity adjustments and measures changes as introduced by Plesser (2003).

Section 3 establishes the link between various classes of interest rate models. This link is useful for a better understand of what follows, but is particularly important for comparison purposes with other studies, where different modeling approaches are used. These sections may be seen as introductory.

Section 4 is the core section of this study. All results concerning convexity adjustments for ATS models are derived and discussed. We start from defining our affine set up. Then, we separate the convexity adjustment we are going to analyze in two categories: forward adjustments and swap adjustments. In the context of forward ajustments, we compute exact formulas for LIBOR in arrears. As an additional example, we also compute the "convexity adjustment" linked to the expectation hypohesis. When dealing with swap convexity adjustments, we base our analysis on Schrager and Pelsser (2006). They show that in an affine term structure setting and under certain common assumptions, the dynamics of the swap rate can be approximated as affine. We use this to derive CMS adjustments in the same spirit as the LIA adjusment. 


\section{Convexity adjustment connection to measure changes}

To give a formal definition of convexity adjustment we need to recall the basics of arbitrage pricing. We start by recalling that in a stochastic interest rate setting, the no arbitrage price $\pi$, at time $t$, of any derivative paying $\Phi(T)$ at maturity $T$ is given by

$$
\pi(t, \Phi)=\mathbb{E}_{t}^{Q}\left[e^{-\int_{t}^{T} r_{u} d u} \Phi(T)\right]=p(t, T) \mathbb{E}_{t}^{T}[\Phi(T)]
$$

where $p(t, T)$, denotes the price at time $t$ of a pure discount bond with maturity $T$ and $\mathbb{E}_{t}^{Q}[\cdot], \mathbb{E}_{t}^{T}[\cdot]$, denote expectation under the risk neutral and the $T$-forward measure, respectively, conditional on the information available at time $t$.

When dealing with fixed income derivatives, given the nature of the underlying asset, we are in a stochastic interest rate setting by definition. Consequently we are ultimately interested in computing, $\mathbb{E}_{t}^{T}[\Phi(T)]$, i.e. the expected value, under the $T$-forward measure where the numeraire is $p(t, T)$, of our payoff.

In the trivial cases, when our payoff turns out to be a $T$ - forward martingale, we immediately get $\mathbb{E}_{t}^{T}[\Phi(T)]=\Phi(t)$. Unfortunately, this is not the case in most situations.

Even if our payoff is not a martingale under the appropriate $T$-forward measure, it could be a martingale under some martingale measure, $\mathbb{Q}^{\Phi}$. This is obviously a restrictive assumption, but as it turns out, it includes many popular interest rate products. It is important to emphasize at this stage that the martingale measure, $\mathbb{Q}^{\Phi}$ is payoff dependent. So, different payoffs are, most likely martingales under different martingale measures.

Suppose, therefore, our payoff is a martingale under some $\mathbb{Q}^{\Phi}$ martingale measure. Then we know $\Phi(t)=\mathbb{E}_{t}^{\Phi}[\Phi(T)]$, and we can define the convexity adjustment as the term $\mathrm{CC}^{\Phi}$ for which the following holds

$$
\mathbb{E}_{t}^{T}[\Phi(T)]=\Phi(t)+\mathrm{CC}^{\Phi}(t)
$$

From the martingale properties of our payoff we get,

$$
\Phi(t)=\mathbb{E}_{t}^{\Phi}[\Phi(T)]=\mathbb{E}_{t}^{T}[\Phi(T) \Lambda(T)]
$$

where $\Lambda$ denotes the Radon-Nikodym derivative (with $\Lambda(t)=1$ ).

Clearly, the cases where we can say something about $\mathbb{E}_{t}^{T}[\Phi(T) \Lambda(T)]$ are the cases where we can say something about convexity adjustment term $\mathrm{CC}^{\Phi}(t)$, which is clearly nothing but a consequence of measure changes.

Using the above mentioned properties one easily derive alternative ways to look into convexity adjustments as

$$
\begin{aligned}
& \mathrm{CC}^{\Phi}(t)=\mathbb{E}_{t}^{T}[\Phi(T)\{1-\Lambda(T)\}] \\
& \mathrm{CC}^{\Phi}(t)=\mathbb{E}_{t}^{T}[\Phi(T)]-\mathbb{E}_{t}^{\Phi}[\Phi(T)] \\
& \mathrm{CC}^{\Phi}(t)=\mathbb{E}_{t}^{T}[\Phi(T)]-\Phi(t)
\end{aligned}
$$

are all equivalent expressions.

If $\mathrm{CC}^{\Phi}$ can be derived explicitly, then all the above $\mathbb{E}_{t}^{T}[\cdot]$ expectations can and vice versa, i.e. if we can obtain one of the above $\mathbb{E}_{t}^{T}[\cdot]$ expectations then we get $\mathrm{CC}^{\Phi}$ as well as the remaining $\mathbb{E}_{t}^{T}[\cdot]$ expectations. 
The way one prefers to "see" the convexity adjustments may vary depending on the exact $\mathbb{Q}^{\Phi}$ martingale measure we have to deal with. Still, at first one may fear nothing much more useful could be said about $\mathrm{CC}^{\Phi}$.

Luckily, as we will see, for many popular interest rate products, there is much more one can say, at least for the ATS class of models.

\section{Linking different Interest Rate Models}

In this section we present the connections between various interest rate model approaches. Concretely, we study the link between short rate models, forward rate models and the so-called market models of interest rate.

Many of the convexity adjustments we will derive are associated with products whose underlying are LIBOR or swap rates. Traditionally, the way to deal with these type of convexities has been to model the underlying rate directly (under the appropriate measure). Thus, most papers have used the market models approach, choosing LIBOR market models or swap market models to model interest rates, depending on the concrete adjustment under analysis.

The problem with choosing a different model to deal with each convexity adjustment is the lack of consistency between the various models. After all, these products are fixed income products and, therefore, one interest rate model should suffice to model all "convexities". Another way to understand this statement is to say that, by choosing a specific, say, swap market model, one is no longer free to choose a LIBOR market model as there are strong no arbitrage relationships between the parameters of both models.

In this study we will use a non market model approach to study convexity adjustments. By using ATS models we are taking a short rate modeling approach. Still, our results can be easily transposed into any other modeling approach provided the connections between the various approaches are clear.

The easiest way to understand all links is to think in terms of pure discount (zero-coupon) bond price dynamics. As we will see, most standard payoffs in interest rate derivatives can be decomposed in terms of two types of martingales: martingales under either some forward measure, or martingales under some swap measure.

Forward measures have as numeraire a pure discount bond with a particular maturity, while swap measures have as numeraire a portfolio of pure discount bonds with different maturities. In the following we explore the relations between the dynamics of discount bonds and those of LIBOR or swap market models. In the following we will refer to pure discount bond prices, simply as bond prices.

Our key dynamics will be the bond prices dynamics under the risk-neutral $\mathbb{Q}$

$$
d p(t, T)=r_{t} p(t, T) d t+v(t, T) p(t, T) d W_{t}
$$

where $r$ is the short interest rate and $v$ is any adapted process.

Most of the results presented in the next subsection have been previously derived elsewhere and are presented without proof. The way we present them should suffice for the reader to follow the arguments and be acquainted with our notation. For proof of the results here presented and further reading on approaches to interest rate modeling we refer to the books James and Webber (2000), Pelsser (2000), Björk (2004) or Brigo and Mercurio (2006). 


\subsection{Forward martingale measures, HJM and LIBOR market models}

Given the bond prices dynamics in (3) we start by looking at instantaneous forward rates. We will denote the forward rate valid at time $t$ for an instantaneous investment at time $T$, by $f(t, T)$. The relationship between bond prices and forwad rates is given by

$$
f(t, T)=-\frac{\partial p(t, T)}{\partial T} \quad \text { or } \quad p(t, T)=\exp \left\{-\int_{t}^{T} f(t, s) d s\right\}
$$

It is well-known ${ }^{1}$ that given the bond price dynamics in $(3)$ the $\mathbb{Q}$-dynamics of forward rates are given by

$$
d f(t, T)=v_{T}(t, T) v(t, T)^{*} d t-v_{T}(t, T) d W_{t} .
$$

Forward rates with maturity $T$ are also $\mathbb{Q}^{T}$ martingales which can be easily understood from their $\mathbb{Q}^{T}$ dynamics,

$$
d f(t, T)=-v_{T}(t, T) d W_{t} .
$$

If we are interested in knowing their dynamics under a different $\mathbb{Q}^{U}$ forward measure (with $U \neq T)$, we can easily obtain

$$
d f(t, T)=v_{T}(t, T)^{*}[v(t, T)-v(t, U)] d t-v_{T}(t, T) d W_{t}^{U} .
$$

We consider now LIBOR rates. By no arbitrage the following relations must hold between forward LIBOR rates and bond prices

$$
L(t, T, S)=\frac{1}{S-T} \frac{p(t, T)-p(t, S)}{p(t, S)} \quad \text { or } \quad \frac{p(t, T)}{p(t, S)}=1+(S-T) L(t, T, S),
$$

where w.l.o.g we take $S \geq T$. Spot LIBOR rates $L(t, S)$ are defined in terms of the forward ones as $L(t, t, S)=L(t, S)$ and, obviously, we have

$$
L(t, S)=\frac{1}{S-t} \frac{1-p(t, S)}{p(t, S)} .
$$

From (6) we can conclude that the forward LIBOR rate with maturity $S$ is a $\mathbb{Q}^{S}$-martingale (i.e. a martingale under the $S$-forward measure). ${ }^{2}$ Also, from (6) and the dynamics of bond prices in (3) we can easily derive the LIBOR rate volatility from the bond prices volatilities. Both things taken together allow us to write the LIBOR rate $\mathbb{Q}^{S}$-dynamics :

$$
d L(t, T, S)=\frac{1}{S-T} \frac{p(t, T)}{p(t, S)}[v(t, T)-v(t, S)] d W_{t}^{S} .
$$

To see the LIBOR dynamics in terms of bond prices is not common in the literature, where people are used to thing in terms of LIBOR markets models. The connection between both approaches is straightforward as we show in Remark 3.1. In our opinion, to think in terms of bond prices is a simpler and more general.

\footnotetext{
${ }^{1}$ See for instance Björk (2004) page 306.

${ }^{2}$ We note that the spot LIBOR rate $L(t, S)$ is not a martingale under the $S$-forward measure. In fact, from (7) and using Itô, we can easily derive $\mathbb{Q}^{S}$-dynamics

$$
d L(t, S)=\left\{\frac{L(t, S)}{S-t}-\frac{1}{S-t} \frac{r_{t}}{p(t, S)}\right\} d t-\frac{v(t, S)}{(S-t) p(t, S)} d W_{t}^{S}
$$
}

and check it has non-zero drift. 


\section{Remark 3.1. (LIBOR market models)}

A popular way to model (forward) LIBOR rates is to assume their dynamics are of the form

$$
d L(t, T, S)=\sigma_{L}(t, T, S) L(t, T, S) d W_{t}^{S}
$$

where $\sigma_{L}(t, S, T)$ is assumed to be either constant or at most a deterministic function of $(t, S, T)$. The usefulness of this approach is that the formulas of Black (1976) are applicable to price caps and floors. This is the case of the so-called LIBOR market models. The connection between (8) and (9) straightforward,

$$
\sigma_{L}(t, T, S)=\frac{p(t, T)}{p(t, T)-p(t, S)}[v(t, T)-v(t, S)] .
$$

We note, however, that assuming (10) deterministic has strong implication on the form of bond prices term structure.

We also note that the forward LIBOR $L(t, T, S)$ is the $\mathbb{Q}^{S}$ expected value, at time $t$, of spot LIBOR rate, valid at time $T$ and over the period $[T, S]$. This results is trivially obtained from the martingale property of the forward LIBOR;

$$
\mathbb{E}_{t}^{S}[L(T, S)]=\mathbb{E}_{t}^{S}[L(T, T, S)]=L(t, T, S) .
$$

Whenever, we are interested in the dynamics of forward LIBOR rates under a forward measure different then their maturity, they are no longer martingales. Still using the change of numeraire technique we can easily obtain their dynamics under $\mathbb{Q}^{U}, U \neq S$ :

$$
d L(t, T, S)=\frac{1}{S-T} \frac{p(t, T)}{p(t, S)}\left\{[v(t, T)-v(t, S)][v(t, S)-v(t, U)]^{*} d t+[v(t, T)-v(t, S)] d W_{t}^{U}\right\}
$$

\subsection{Swap martingale measures and swap market models}

We, now look at (forward) swap rates. We consider a standard fixed-for-floating swap with payment dates at $T_{1}, \cdots, T_{N}$. We assume the floating leg of the swap will receive at each time $T_{i}$ the LIBOR rate $L\left(T_{i-1}, T_{i}\right)$ (observed at time $T_{i-1}$ ) while the fixed leg will receive a fixed rate $S\left(t, T_{0}, T_{N}\right)$ decided at time $t$ (with $t \leq T_{0}$ ). $T_{0}$ is, thus, the starting date of the first effective period of the swap, $t$ is the contractual date and the swap rate $S\left(t, T_{0}, T_{N}\right)$ is decided so that the swap contract is worth zero at time $t$. Finally, we define $\alpha_{i}=T_{i}-T_{i-1}$.

It is quite straight forward to see that the value, at time $t$, of the floating leg of the swap is

$$
\begin{aligned}
\sum_{i=1}^{N} p\left(t, T_{i}\right) \alpha_{i} \mathbb{E}_{t}^{T_{i}}\left[L\left(T_{i-1}, T_{i}\right)\right] & =\sum_{i=1}^{N} p\left(t, T_{i}\right) \alpha_{i} \mathbb{E}_{t}^{T_{i}}\left[L\left(T_{i-1}, T_{i-1}, T_{i}\right)\right] \\
& =\sum_{i=1}^{N} p\left(t, T_{i}\right) \alpha_{i} L\left(t, T_{i-1}, T_{i}\right) \\
& =\sum_{i=1}^{N} p\left(t, T_{i}\right) \frac{p\left(t, T_{i-1}\right)-p\left(t, T_{i}\right)}{p\left(t, T_{i}\right)} \\
& =p\left(t, T_{0}\right)-p\left(t, T_{N}\right)
\end{aligned}
$$


On the other hand, the fixed leg is worth $\sum_{i=1}^{N} p\left(t, T_{i}\right) \alpha_{i} S\left(t, T_{0}, T_{N}\right)$ hence we get

$$
S\left(t, T_{0}, T_{N}\right)=\frac{p\left(t, T_{0}\right)-p\left(t, T_{N}\right)}{\sum_{i=1}^{N} \alpha_{i} p\left(t, T_{i}\right)} .
$$

The swap rate $S\left(t, T_{0}, T_{N}\right)$ is a martingale under the equivalent martingale measure where the numeraire is the portfolio $P$ of discount bonds :

$$
P\left(t, T_{0}, T_{N}\right)=\sum_{i=1}^{N} \alpha_{i} p\left(t, T_{i}\right) .
$$

The measure with such a numeraire is usually called the swap measure and we will denote it by $\mathbb{Q}^{\mathcal{S}}$. This measure depends strongly on the payment dates $T_{1}, \cdots, T_{N}$, so it specific of each swap contract.

The portfolio $P$ dynamics are defined only until $T_{1}$ (the lowest maturity of the bonds in the portfolio). Under the risk-neutral measure $\mathbb{Q}$, we have

$$
d P\left(t, T_{0}, T_{N}\right)=r P\left(t, T_{0}, T_{N}\right) d t+V\left(t, T_{0}, T_{N}\right) P\left(t, T_{0}, T_{N}\right) d W_{t}
$$

where $V$ is simply $V\left(t, T_{0}, T_{N}\right)=\frac{\sum_{i=1}^{N} \alpha_{i} p\left(t, T_{i}\right) v\left(t, T_{i}\right)}{P\left(t, T_{0}, T_{N}\right)}$.

Since the swap rate $S\left(t, T_{0}, T_{N}\right)$ is a martingale under the swap measure we can write its $\mathbb{Q}^{\mathcal{S}}$-dynamics as

$$
d S\left(t, T_{0}, T_{N}\right)=\delta\left(t, T_{0}, T_{N}\right) d W_{t}^{\mathcal{S}}
$$

with

$$
\delta\left(t, T_{0}, T_{N}\right)=\frac{v\left(t, T_{0}\right) p\left(t, T_{0}\right)-v\left(t, T_{N}\right) p\left(t, T_{N}\right)-\left[p\left(t, T_{0}\right)-p\left(t, T_{N}\right)\right] V\left(t, T_{0}, T_{N}\right)}{P\left(t, T_{0}, T_{N}\right)} .
$$

\section{Remark 3.2. (Swap market models)}

A popular way to model (forward) swap rates is to assume their dynamics are of the form

$$
d S\left(t, T_{0}, T_{N}\right)=\sigma_{S}\left(t, T_{0}, T_{N}\right) S\left(t, T_{0}, T_{N}\right) d W_{t}^{\mathcal{S}}
$$

where $\sigma_{S}\left(t, T_{0}, T_{N}\right)$ is assumed to be either constant or at most a deterministic function. The usefulness of this approach is that the formulas of Black (1976) are applicable to price swaptions. Comparing the swap dynamics in (11)-(12) with the dynamics in (13), one can easily derive

$$
\begin{aligned}
\sigma_{S}\left(t, T_{0}, T_{N}\right) & =\frac{v\left(t, T_{0}\right) p\left(t, T_{0}\right)-v\left(t, T_{N}\right) p\left(t, T_{N}\right)}{p\left(t, T_{0}\right)-p\left(t, T_{N}\right)}-V\left(t, T_{0}, T_{N}\right) \\
& =\frac{v\left(t, T_{0}\right) p\left(t, T_{0}\right)-v\left(t, T_{N}\right) p\left(t, T_{N}\right)}{p\left(t, T_{0}\right)-p\left(t, T_{N}\right)}-\frac{\sum_{i=1}^{N} \alpha_{i} p\left(t, T_{i}\right) v\left(t, T_{i}\right)}{P\left(t, T_{0}, T_{N}\right)}
\end{aligned}
$$

We emphasize that assuming (15) deterministic has strong implication on the form of bond prices term structure.

Given the $\mathbb{Q}^{\mathcal{S}}$-dynamics in (11)-(12), under any $\mathbb{Q}^{U}$-forward measure we obtain

$$
d S\left(t, T_{0}, T_{N}\right)=\delta\left(t, T_{0}, T_{N}\right)\left[V\left(t, T_{0}, T_{N}\right)-v(t, U)\right]+\delta\left(t, T_{0}, T_{N}\right) d W_{t}^{U} .
$$




\section{Convexity Adjustments for ATS models}

We will consider affine term structure (ATS) models. This setup assumes as given factors described by a $\mathbb{R}^{m}$-valued stochastic process $\left(Z_{t}\right)_{t \geq 0}$ whose dynamics, under the risk-neutral martingale measure $\mathbb{Q}$, are of a special form. Furthermore, it assumes that the risk-free rate of interest $r$ is affine on those factors.

Assumption 4.1. $W$ is an n-dimensional standard Brownian motion and $Z$ is the unique strong solution of

$$
d Z_{t}=\alpha\left(t, Z_{t}\right) d t+\sigma\left(t, Z_{t}\right) d W_{t}
$$

Here $\alpha: \mathbb{R}_{+} \times \mathbb{R}^{m} \mapsto \mathbb{R}^{m}$ and $\sigma: \mathbb{R}_{+} \times \mathbb{R}^{m} \mapsto \mathbb{R}^{n \times n}$ are such that

$$
\begin{aligned}
\alpha(t, z) & =d(t)+E(t) z \\
\sigma(t, z) \sigma^{\top}(t, z) & =k_{0}(t)+\sum_{i=1}^{m} k_{i}(t) z_{i}
\end{aligned}
$$

with smooth functions $d: \mathbb{R}_{+} \mapsto \mathbb{R}^{m}, E, k_{0}, k_{i} i=1, \cdots, m$ map $\mathbb{R}_{+}$to $\mathbb{R}^{m \times m}$. Moreover, the risk-free short rate $\left(r_{t}\right)_{t \geq 0}$ is given by

$$
r\left(t, Z_{t}\right)=f(t)+g(t)^{*} Z_{t} .
$$

$f, g$ are smooth, mapping $\mathbb{R}_{+}$to $\mathbb{R}^{m}$ and $\mathbb{R}$, respectively.

In this context, we know that bond prices can be written as

$$
p(t, T)=\exp \left\{A(t, T)+B(t, T)^{*} Z_{t}\right\}
$$

where $A, B$ are deterministic functions of $(t, T)$ and solve the ODE system,

$$
\begin{aligned}
\frac{\partial A}{\partial t}+d(t)^{*} B+\frac{1}{2} B^{*} k_{0}(t) B & =f(t) \\
\frac{\partial B}{\partial t}+E(t)^{*} B+\frac{1}{2} \bar{B}^{*} K(t) B & =g(t)
\end{aligned}
$$

subject to the boundary conditions $A(T, T)=0$ and $B(T, T)=0$. $A$ and $B$ should always be evaluated at $(t, T) . E, d, k_{0}$, are from (17)-(18) and $f, g$ from (19) while

$$
\bar{B}:=\left(\begin{array}{cccc}
B & 0 & \cdots & 0 \\
0 & B & \cdots & 0 \\
\vdots & & \ddots & \\
0 & \cdots & 0 & B
\end{array}\right), \quad K(t)=\left(\begin{array}{c}
k_{1}(t) \\
\vdots \\
k_{m}(t)
\end{array}\right) .
$$

Applying Itô to (20), one can easily rewrite $\mathbb{Q}$-bond dynamics as

$$
d p(t, T)=r_{t} p(t, T) d t+B(t, T)^{*} \sigma\left(t, Z_{t}\right) p(t, T) d W .
$$

So in particular by comparing the above expression with (3) we get $v(t, T)=B(t, T)^{*} \sigma\left(t, Z_{t}\right)$.

These are well-known results from the ATS literature and can be found in textbooks. We refer to Duffie, Pan, and Singleton (2000) and Duffie, Filipović, and Schachermayer (2003). 
The class of ATS has also been extended to general quadratic term structures GQTS by Gaspar (2004) and Cheng and Scaillet (2007). The class of GQTS is more general than the ATS one, but the question of how much more general is still an open debate. For simplicity and because the market practice is to use ATS model we restrict our analysis to that case. Extending all results presented in the next subsections to the GQTS case would be cumbersome but rather straightforward exercise.

\subsection{Forward Convexity Adjustments}

\subsubsection{Expectation Hypothesis adjustment}

Consider a derivative that at some time $T$ in the future we will pay you the instantaneous short rate, $r(T)$, on the notional 1 euro. Its value today, $\pi(t)$, is given by

$$
\begin{aligned}
\mathbb{E}_{t}^{\mathbb{Q}}\left[\exp \left\{-\int_{t}^{T} r(u) d u\right\} r(T)\right] & =\mathbb{E}_{t}^{\mathbb{Q}}\left[\exp \left\{-\int_{t}^{T} r(u) d u\right\} f(T, T)\right] \\
& =p(t, T) \mathbb{E}_{t}^{T}[f(T, T)] \\
& =p(t, T) f(t, T)
\end{aligned}
$$

where we have used the fact that $f(t, T)$ is a $\mathbb{Q}^{T}$-martingale.

Consider the same derivative again, but suppose now we only get paid at a later time $S \geq T$ ? Well, at time $T$, the payoff is fully defined and the value at of our derivative must be $\pi(T)=$ $p(T, S) r(T)$ and, thus, at any time $t$ we have

$$
\pi(t)=p(t, S) \mathbb{E}_{t}^{S}[r(T)]
$$

Clearly we see that $\pi(t) \neq p(t, S) \mathbb{E}_{t}^{T}[r(T)]=p(t, S) f(t, T)$, but since both $p(t, S)$ and $f(t, T)$ are information we can directly obtain from the market we may wonder how we can still use this information to get the correct price.

That is, we would like to find $\mathrm{CC}^{E H}(\cdot)$ such that

$$
\pi(t)=p(t, S) f(t, T)\left[1+\mathrm{CC}^{E H}(t, T, S)\right] .
$$

Comparing (25) and (26) we can formally define our first convexity adjustment.

Definition 4.2. The expectation hypothesis adjustment is defined as

$$
\mathrm{CC}^{E H}(t, T, S)=\mathbb{E}_{t}^{S}[r(T)]-f(t, T) .
$$

It is obvious that for $T=S$ the $\operatorname{CC}^{E H}(t, T, S)=0$, i.e., there is no convexity adjustment. It is also clear that the $\mathrm{CC}$ will be further away from 0 the more $\mathbb{E}_{t}^{S}[r(T)]$ differs from the instantaneous forward rate.

Proposition 4.3. Suppose Assumption 4.1 holds. Then, the expectation hypothesis adjustment of Definition 4.2 can be obtained in closed-form and is given by

$$
\mathrm{CC}^{E H}(t, T, S)=F(t, T, S)+G(t, T, S)^{*} Z_{t}
$$


where $F$ and $G$ are deterministic functions of $(t, T, S)$ solving the following ODE system

$$
\begin{aligned}
& \left\{\begin{aligned}
\frac{\partial F}{\partial t}+B_{T}(t, T)^{*} k_{0}(t)[B(t, T)-B(t, S)]+d(t)^{*} G-B(t, S)^{*} k_{0}(t) G & =0 \\
F(T, T, S) & =0
\end{aligned}\right. \\
& \left\{\begin{aligned}
\frac{\partial G}{\partial t}+\bar{B}_{T}(t, T)^{*} K(t)[B(t, T)-B(t, S)]+E(t)^{*} G-\bar{B}(t, S)^{*} K(t) G & =0 \\
G(T, T, S) & =0
\end{aligned}\right.
\end{aligned}
$$

where $F$ and $G$ should always be evaluated at $(t, T, S) . E, d, k_{0}$, are from (17)-(18) and $K, \bar{B}$ are as in (23).

Proof. (sketch)

Provided (28) holds then we have

$$
\mathbb{E}_{t}^{S}[r(T)]=f(t, T)+F(t, T, S)+G(t, T, S)^{*} Z_{t} .
$$

Note the instantaneous forward rates, $f(t, T)$ are fully determined by the system of ODEs (21)(22). To see this note that from the relationship between bond prices and forward prices (recall (4)) and the functional form for bond price in the ATS setup in (20), we get

$$
f(t, T)=-\left(A_{T}(t, T)+B_{T}^{*}(t, T) Z_{t}\right)
$$

where $B_{T}$ stands for applying $\frac{\partial}{\partial T}$ to each cell in $B$, while $A_{T}=\frac{\partial A}{\partial T}$, with $A, B$ as in (21)-(22). Define $M(t, T, S)=\mathbb{E}_{t}^{S}[r(T)]$, which by definition is a $\mathbb{Q}^{S}$-martingale. Using the assumed functional, one can see $M$ as a deterministic function of two stochastic variables $Z$ and $f(\cdot, T)$,

$$
M\left(t, Z_{t}, f(t, T), T, S\right)=f(t, T)+F(t, T, S)+G(t, T, S)^{*} Z_{t}
$$

To be able to apply Itô and get our result we need the dynamics of both $Z$ and $f(\cdot, T)$ under $\mathbb{Q}^{S}$. From the $\mathbb{Q}$-dynamics of $Z$ in Assumption 4.1, we can easily derive its $\mathbb{Q}^{S}$ dynamics as

$$
d Z_{t}=\left[\alpha\left(t, Z_{t}\right)-v(t, S) \sigma\left(t, Z_{t}\right)^{*}\right] d t+\sigma\left(t, Z_{t}\right) d W_{t}^{S} .
$$

with $\alpha, \sigma$ in (17)-(18). From (5) we obtain $\mathbb{Q}^{S}$ dynamics of $f(\cdot, T)$,

$$
d f(t, T)=v_{T}(t, T)^{*}[v(t, T)-v(t, S)] d t-v_{T}(t, T) d W_{t}^{S} .
$$

Using Itô and its martingale property, one easily obtain the following PDE, $M$ must satisfy

$$
\left\{\begin{aligned}
\frac{\partial M}{\partial t}+\frac{\partial M}{\partial f} v_{T}(t, T)[v(t, T)-v(t, S)]^{*}+\frac{\partial M}{\partial z} G\left[\alpha\left(t, Z_{t}\right)-v(t, S) \sigma\left(t, Z_{t}\right)^{*}\right] & =0 \\
M(T, T, S) & =f(T, T)
\end{aligned}\right.
$$

Computing all partial derivatives and using $v(t, T)=B(t, T)^{*} \sigma\left(t, Z_{t}\right)$, we can easily re-write our PDE in terms of $F$ and $G$

$$
\left\{\begin{aligned}
\frac{\partial F}{\partial t}+\frac{\partial G}{\partial t} Z_{t}+B_{T}(t, T)^{*} \Sigma\left(t, Z_{t}\right)[B(t, T)-B(t, S)] & \\
+G\left[d(t)+E(t) Z_{t}-B(t, S)^{*} \Sigma\left(t, Z_{t}\right)\right] & =0 \\
F(T, T, S)+G(T, T, S) Z_{t} & =0
\end{aligned}\right.
$$

with $\Sigma(t, z)$ as in (18). We can now use separation of variables and identify terms to obtain the system of ODEs in (29)-(30). 


\subsubsection{LIA adjustment}

Standard products whose payoff depend upon LIBOR rates (like vanilla interest rate swaps, for instance), typically involve payment of LIBOR rates $L(T, S)$ at the end of the effective period they apply to, that is at $S$, despite the fact that they are actually determined at time $T$. There are, however, more exotic products that may require payments in arrears, that is payoffs that would depend on $L(T, S)$ would be paid out at the beginning of the period the LIBOR apply to, that is at time $T$. An example of such products is the LIBOR in arrears swap.

Recall from Section 3 that the forward LIBOR rate $L(t, T, S)$ can be expressed in terms of discount bond prices as

$$
L(t, T, S)=\frac{1}{\alpha} \frac{p(t, T)-p(t, S)}{p(t, S)},
$$

where $\alpha=S-T$. Also, spot LIBOR rates are defined as $L(t, S)=L(t, t, S)$. From the above expression one can easily see the forward LIBOR rate $L(t, T, S)$ is a martingale under the forward martingale measure $\mathbb{Q}^{S}$. Thus, to price future LIBOR depended payments due at $S$, we have $\mathbb{E}_{t}^{S}[L(T, S)]=\mathbb{E}_{t}^{S}[L(T, T, S)]=L(t, T, S)$ and get

$$
\mathbb{E}_{t}^{\mathbb{Q}}\left[e^{-\int_{t}^{S} r(u) d u} L(T, S)\right]=p(t, S) \mathbb{E}_{t}^{S}[L(T, T, S)]=p(t, S) L(t, T, S) .
$$

This is the case, for instance, in standard swap contracts.

When pricing in-arrears products, however, we are interested in computing expectations of the form

$$
\mathbb{E}_{t}^{\mathbb{Q}}\left[e^{-\int_{t}^{T} r(u) d u} L(T, S)\right]=p(t, T) \mathbb{E}_{t}^{T}[L(T, S)]=p(t, T) \mathbb{E}_{t}^{T}[L(T, T, S)] .
$$

As forward LIBOR rates are martingales under the $S$ - forward measure and not the $T$-forward measure. We know $\mathbb{E}_{t}^{T}[L(T, T, S)] \neq L(t, T, S)$.

In the same spirit as before, we can define the arreas convexity adjustment as

$$
\mathbb{E}_{t}^{T}[L(T, S)]=L(t, T, S)+\mathrm{CC}^{L I A}(t, T, S) .
$$

For this particular convexity adjustment, we note the measure change we are interested in occurs between two forward measures. In this case we know

$$
\mathbb{E}_{t}^{T}\left[L(T, S]=\mathbb{E}_{t}^{S}\left[L(T, S) \frac{1+\alpha L(T, S)}{1+\alpha L(t, T, S)}\right]\right.
$$

which implies

$$
\mathrm{CC}^{L I A}(t, T, S)=\alpha \frac{\mathbb{E}^{S}\left[(L(T, S))^{2}\right]-[L(t, T, S)]^{2}}{1+\alpha L(t, T, S)}=\frac{\alpha \operatorname{Var}_{t}[L(T, S)]}{1+\alpha L(t, T, S)}
$$

This is valid irrespective of the distribution we assume for the forward LIBOR rates. Moreover, it only depends on its variance which is the same under any equivalent martingale measure.

\section{Remark 4.4. Lognormal LIBOR rates}

If we are in the context of LIBOR market models, where LIBOR rates are assumed to be lognormaly distributed, one can easily obtain closed-form solutions. Indeed,

$$
\mathrm{CC}^{L I A}(t, T, S)=\frac{\alpha(L(t, T, S))^{2} e^{\sigma_{L}^{2}(T-t)}}{1+\alpha L(t, T, S)}
$$

where $\sigma_{L}$ represents the LIBOR volatility as in (10) taken as constant. 
For further details on the convexity adjustment for lognomal LIBOR rates we refer to $\mathrm{Pu}$ gachevsky (2001).

More generally, we can define an adjustment for when we take expectations of the LIBOR rates under any other $U$-forward measure, such that $T \leq U \leq S$.

Definition 4.5. The LIBOR in arrears adjustment is defined as

$$
\mathrm{CC}^{L I A}(t, T, U, S)=\mathbb{E}_{t}^{U}[L(T, S)]-L(t, T, S) .
$$

Proposition 4.6. Suppose Assumption 4.1 holds. Then, the LIBOR in arrears adjustment of Definition 4.5 can be obtained in closed-form and is given by

$$
\mathrm{CC}^{L I A}(t, T, U, S)=\frac{1}{S-T} \frac{p(t, T)}{p(t, S)}\left[e^{F(t, T, U, S)+G(t, T, U, S) Z_{t}}-1\right]
$$

where $F$ and $G$ are deterministic functions of $(t, T, U, S)$ and solve the following ODE system,

$$
\begin{gathered}
\left\{\begin{aligned}
\frac{\partial F}{\partial t}+[B(t, S)-B(t, U)]^{*} k_{0}(t)[B(t, T)-B(t, S)]+d(t)^{*} G-B(t, U)^{*} k_{0}(t) G & \\
+\frac{1}{2} G^{*} k_{0}(t) G+[B(t, T)-B(t, S)]^{*} k_{0}(t) G & =0 \\
F(T, T, U, S) & =0
\end{aligned}\right. \\
\left\{\begin{aligned}
\frac{\partial G}{\partial t}+[\bar{B}(t, S)-\bar{B}(t, U)]^{*} K(t)[B(t, T)-B(t, S)]+E(t)^{*} G-\bar{B}(t, U) K(t) G & \\
+\frac{1}{2} \bar{G}^{*} K(t) G+[\bar{B}(t, T)-\bar{B}(t, S)]^{*} K(t) G & =0 \\
G(T, T, U, S) & =0
\end{aligned}\right.
\end{gathered}
$$

where where $F$ and $G$ should always be evaluated at $(t, T, U, S) . E, d, k_{0}$, are from (17)-(18) and $K, \bar{B}$ are as in (23).

Proof. (sketch)

We start by noting that, given the relationship between LIBOR rates and bond prices in (6), we have

If (33) holds, it follows that

$$
\mathbb{E}_{t}^{U}[L(T, S)]=\frac{1}{S-T}\left[\mathbb{E}_{t}^{U}\left[\frac{1}{p(T, S)}\right]-1\right] .
$$

$$
1+(S-T) \mathbb{E}_{t}^{U}[L(T, S)]=\mathbb{E}_{t}^{U}\left[\frac{1}{p(T, S)}\right]=\mathbb{E}_{t}^{U}\left[\frac{p(T, T)}{p(T, S)}\right]=\frac{p(t, T)}{p(t, S)} e^{F(t, T, U, S)+G(t, T, U, S) Z_{t}}
$$

Also note $Y_{t}=\frac{p(t, T)}{p(t, S)}$ is fully determined by the ODE system of equations in (21)-(22) and its is a $\mathbb{Q}^{S}$-martingale. Thus, its $\mathbb{Q}^{U}$-dynamics are given by

$$
d Y_{t}=[v(t, S)-v(t, U)][v(t, T)-v(t, S)]^{*} Y_{t} d t+[v(t, T)-v(t, S)] Y_{t} d W_{t}^{U} .
$$

Let us now define $M(t, T, U, S)=\mathbb{E}_{t}^{U}\left[\frac{p(T, T)}{p(T, S)}\right]$. Note $M$ is a $\mathbb{Q}^{U}$-martingale. Provided $M\left(t, Z_{t}, Y_{t} ; T, U, S\right)=Y_{t} e^{F(t, T, U, S)+G(t, T, U, S) Z_{t}}$ holds, we can see $M$ as specific function of two stochastic processes: $Y$ and $Z$. From (31) we also know

$$
d Z_{t}=\left[\alpha\left(t, Z_{t}\right)-v(t, U) \sigma\left(t, Z_{t}\right)\right] d t+\sigma\left(t, Z_{t}\right) d W_{t}^{U} .
$$


Using Itô infinitesimal operator on $M$ and the fact $M$ is a $\mathbb{Q}^{U}$ martingale we get the PDE

$$
\begin{gathered}
\frac{\partial M}{\partial t}+\frac{\partial M}{\partial y}[v(t, S)-v(t, U)][v(t, T)-v(t, S)]^{*} y+\frac{\partial M}{\partial z}[\alpha(t, z)-v(t, U) \sigma(t, z)]+ \\
+\frac{1}{2} \frac{\partial^{2} M}{\partial z^{2}} \sigma(t, z) \sigma(t, z)^{*}+\frac{\partial^{2} M}{\partial z \partial y}[v(t, T)-v(t, S)] \sigma(t, z)^{*}=0
\end{gathered}
$$

where all partial derivative of $M$ should be evaluated at $\left(t, Z_{t}, Y_{t} ; T, U, S\right)$. Also we have for $t=T: M(T ; T, U, S)=y$.

From the functional form of $M$ we can compute all partial derivatives and transform our PDE into an ODE

$$
\begin{gathered}
\left(\frac{\partial F}{\partial t}+\frac{\partial G}{\partial t}\right) M+[v(t, S)-v(t, U)][v(t, T)-v(t, S)]^{*} M+G\left[\alpha\left(t, Z_{t}\right)-v(t, U) \sigma\left(t, Z_{t}\right)\right] M+ \\
+\frac{1}{2} G \sigma\left(t, Z_{t}\right) G+G[v(t, T)-v(t, S)] \sigma\left(t, Z_{t}\right)^{*}=0
\end{gathered}
$$

or $F(T ; T, U, S)=0$ and $G(T ; T, U, S)=0$. Finally, using the affine dynamics of $Z$ and separating variables we obtain the system of ODEs in (34)-(35)

\subsection{Swap Convexity Adjustments}

Since interest rate swaps make up a big proportion of the OTC derivatives market, convexity adjustments needed to price non standard products whose underlying are swap rates are particularly important.

We start by recalling from Section 3.2 that the forward swap rate $S\left(t, T_{0}, T_{N}\right)$ quoted at time $t$, with start date $T_{0}$ and maturity $T_{N}$ is model-free and equal to

$$
S\left(t, T_{0}, T_{N}\right)=\frac{p\left(t, T_{0}\right)-p\left(t, T_{N}\right)}{P\left(t, T_{0}, T_{N}\right)},
$$

where $P\left(t, T_{0}, T_{N}\right)=\sum_{i=1}^{N} \alpha_{i} p\left(t, T_{i}\right)$ for $\alpha_{i}$ is known as swap level. Swap rates are, thus. martingales under the so-called $\mathbb{Q}^{\mathcal{S}}$ swap martingale measure, whose numeraire is the swap level. This measure is specific of each swap as it depends on the payments tenor structure. Finally, recall that by no arbitrage arguments, one can always write any swap rate dynamics in terms of bond prices and their volatility as in (11)-(12).

In the context of ATS models, Schrager and Pelsser (2006) suggest an approximation to the volatility of swap rates based upon a so-called low variance assumption.

\section{Assumption 4.7. (Low Variance)}

We assume $\frac{p\left(t, T_{i}\right)}{P\left(t, T_{0}, T_{N}\right)}$, for all $i=1, \cdots, T_{N}$, are low variance $\mathbb{Q}^{\mathcal{S}}$-martingales.

The low variance assumption is not unusual in the context of obtaining swap rate volatilities and has been used before in the context of LIBOR market models. Brace and R.Womersley (2000) investigate thoroughly the satistical consequences of making such an assumption and find that the resulting approximative dynamics for swaps work well in the context of LMM.

Schrager and Pelsser (2006) claim this property should holds in general and investigate its consequences in the context of affine term structure models. To us it is still unclear why it should hold in real life. More importantly we would like to understand its implications in 
terms of restrictions on the shape of the bond prices term structure. From a mathematical point of view it is, without question a useful assumption. Assuming $\frac{p\left(t, T_{i}\right)}{P\left(t, T_{0}, T_{N}\right)}$ are low variance martingales is equivalent to assuming

$$
\frac{p\left(t, T_{i}\right)}{P\left(t, T_{0}, T_{N}\right)} \approx \frac{p\left(0, T_{i}\right)}{P\left(0, T_{0}, T_{N}\right)}
$$

since $\frac{p\left(0, T_{i}\right)}{P\left(0, T_{0}, T_{N}\right)}$ is the conditional expectation, at time $t=0$ of the ratio $\frac{p\left(t, T_{i}\right)}{P\left(t, T_{0}, T_{N}\right)}$ under the swap measure.

Then, clearly, the diffusion term $\delta\left(t, T_{0}, T_{N}\right)$ in (12) can be simplified to

$$
\begin{aligned}
\delta\left(t, T_{0}, T_{N}\right)=\{ & B^{*}\left(t, T_{0}\right) \frac{p\left(0, T_{0}\right)}{P\left(0, T_{0}, T_{N}\right)}-B^{*}\left(t, T_{N}\right) \frac{p\left(0, T_{N}\right)}{P\left(0, T_{0}, T_{N}\right)}- \\
& \left.-S\left(0, T_{0}, T_{N}\right) \sum_{i=1}^{N} \frac{\alpha_{i} p\left(0, T_{i}\right)}{P\left(0, T_{0}, T_{N}\right)} B^{*}\left(t, T_{i}\right)\right\} \sigma\left(t, Z_{t}\right),
\end{aligned}
$$

where $\sigma\left(t, Z_{t}\right)$ is the volatility of the affine factors $Z_{t}$ and is the only stochastic term.

The important point to make here is that this implies that the variance covariance matrix for the swap rate dynamics is also affine and we can re-write the swap dynamics as

$$
d S\left(t, T_{0}, T_{N}\right) \approx\left[\sum_{j=1}^{N} q_{j}(0) B\left(t, T_{j}\right)\right]^{*} \sigma\left(t, Z_{t}\right) d W_{t}^{\mathcal{S}}
$$

where

$$
q_{0}=\frac{p\left(0, T_{0}\right)}{P\left(0, T_{0}, T_{N}\right)}, q_{N}=\frac{p\left(0, T_{N}\right)\left[1+\alpha_{N-1} S\left(0, T_{0}, T_{N}\right)\right]}{P\left(0, T_{0}, T_{N}\right)}, q_{j}=\frac{p\left(0, T_{j}\right) \alpha_{j-1} S\left(0, T_{0}, T_{N}\right)}{P\left(0, T_{0}, T_{N}\right)} .
$$

For simplify, in the following, we will use the notation

$$
Q_{0}\left(t, T_{0}, T_{N}\right)=\sum_{j=1}^{N} q_{j}(0) B\left(t, T_{j}\right)
$$

to denote the deterministic part of the diffusion term in (36)

\subsubsection{CMS adjustment}

A constant maturity swap (CMS) contract exchanges a swap rate with a certain time to maturity $c$ against a fixed payment $K$. In a standard swap, we exchange a LIBOR against a fixed payment. In the CMS, the floating rate is no longer a LIBOR, but a swap rate with a certain time to maturity, denoted here by $c$. For example, we pay every 6 months the 5 year swap rate and receive a fixed payment $K$. By a reasoning similar to the one used in Section 3.2, one can easily derive the formula for the CMS rate $K$,

$$
K=\frac{\sum_{i=1}^{n} \alpha_{i} p\left(t, T_{i}\right) \mathbb{E}_{t}^{T_{i}}\left[S\left(T_{i-1}, T_{i}, T_{i+c}\right)\right]}{\sum_{i=1}^{n} \alpha_{i} p\left(t, T_{i-1}\right)}
$$


The difficulty is in computing the value of each payment of the floating leg, and to be more specific, in computing $\mathbb{E}_{t}^{T_{i}}\left[S\left(T_{i-1}, T_{i}, T_{i+c}\right)\right]$. As $S\left(T_{i-1}, T_{i}, T_{i+c}\right)$ is not a martingale under forward measures, we need a convexity adjustments to take care of this. The convexity adjustment for each payment of a CMS results from

$$
\mathbb{E}_{t}^{T_{i}}\left[S\left(T_{i-1}, T_{i}, T_{i+c}\right)\right]=S\left(t, T_{i}, T_{i+c}\right)+\mathrm{CC}^{C M S}\left(t, T_{i}, c\right)
$$

and relies on measure changes from the $T_{i}$-forward measure to $\mathbb{Q}^{\mathcal{S}}$.

Definition 4.8. The CMS adjustement is defined as

$$
\mathrm{CC}^{C M S}\left(t, T_{i}, c\right)=\mathbb{E}_{t}^{T_{i}}\left[S\left(T_{i-1}, T_{i}, T_{i+c}\right)\right]-S\left(t, T_{i}, T_{i+c}\right) .
$$

Alternatively, one can see this adjustment as

$$
C C^{C M S}\left(t, T_{i}, c\right)=\frac{P\left(t, T_{i}, T_{i+c}\right)}{p\left(t, T_{i}\right)} \mathbb{E}_{t}^{\mathcal{S}}\left[S\left(T_{i-1}, T_{i}, T_{i+c}\right) \frac{p\left(T_{i-1}, T_{i}\right)}{P\left(T_{i-1}, T_{i}, T_{i+c}\right)}\right]-S\left(t, T_{i}, T_{i+c}\right)
$$

where the expectation on the RHS is now taken under the swap measure $\mathbb{Q}^{\mathcal{S}}$.

This is not easy to obtain in closed-form. Most CMS convexity adjustment formulae are only possible to get in an approximative way.

A popular trick, at this stage, is to see $p\left(t, T_{i}\right) / P\left(t, T_{i}, T_{i+c}\right)$ as a function, $G$ of the swap rate $S\left(t, T_{i}, T_{i+c}\right)$ ). In the case of the Linear Swap Model (LSM) introduced by Hunt and Kennedy (2000), $G$ is assumed to be linear in the swap rate $S\left(t, T_{i}, T_{i+c}\right)$. For a generic (truly convex) function $G$, what has been considered so far, are Taylor expansions around today's swap rate $S\left(t, T_{i}, T_{i+c}\right)$. A popular paper on convexity adjustments of CMS swaps which goes along this way is the one by Hagan (2003). The author analyzes three basic term structure models, starting from a standard yield curve model and continuing to more sophisticated models which account for non-parallel shifts and continuous time compounding of interest rates. This is equivalent to assuming particular functional forms for the $G$ function. Given a functional for $G$, he then uses a first order Taylor approximation and a lognormal distribution for the swap rates to get approximations for the CMS convexity adjustment

$$
C C^{C M S}\left(t, T_{i}, c\right) \approx S\left(t, T_{i}, T_{i+c}\right) \frac{G^{\prime}\left(S\left(t, T_{i}, T_{i+c}\right)\right)}{G\left(S\left(t, T_{i}, T_{i+c}\right)\right)}\left[S\left(t, T_{i}, T_{i+c}\right) e^{\sigma^{2}\left(T_{i-1}-t\right)}-1\right],
$$

where $\sigma$ can be taken to be Black (1976) implied volatility from at-the-money vanilla swaptions market quotes. For convexity adjustments of CMS caps and floors we refer to the paper itself. The method proposed in Hagan (2003) includes as special case the LSM, in which case the a first order Taylor approximation is in fact exact.

Here we take different approach and follow the low variance assumption of Schrager and Pelsser (2006). The approximative dynamics of swaps in the affine term structure setup in (36)-(37) are all we need to derive formulae for the CMS convexity adjustment.

Proposition 4.9. Let Assumptions 4.1 and 4.7 hold. Then, the CMS adjustment of Definition 4.8 can be approximated by

$$
\mathrm{CC}^{C M S}\left(t, T_{i}, c\right) \approx F\left(t, T_{i}, T_{i+c}\right)+G\left(t, T_{i}, T_{i+c}\right) Z_{t}
$$


where $F$ and $G$ are deterministic functions of $\left(t, T_{i}, T_{i+c}\right)$ and solve the following ODE system:

$$
\left\{\begin{aligned}
\frac{\partial F}{\partial t}-Q_{0}\left(t, T_{i}, T_{i+c}\right)^{*} k_{0}(t) B\left(t, T_{i}\right)+d(t)^{*} G-B\left(t, T_{i}\right)^{*} k_{0}(t) G & =0 \\
F\left(T_{i}, T_{i}, T_{i+c}\right) & =0 \\
\frac{\partial G}{\partial t}-\bar{B}\left(t, T_{i}\right)^{*} K Q_{0}\left(t, T_{i}, T_{i+c}\right)+E(t)^{*} G-\bar{B}\left(t, T_{i}\right)^{*} K G & =0 \\
G\left(T_{i}, T_{i}, T_{i+c}\right) & =0
\end{aligned}\right.
$$

where $F$ and $G$ should always be evaluated at $\left(t, T_{i}, T_{i+c}\right) . E, d, k_{0}$, are from (17)-(18) $K, \bar{B}$ are as in (23) and $Q_{0}$ is as in (37).

\section{Proof. (sketch)}

The proof of this proposition is very similar in spirit to the previous ones. The object we need to compute is $\mathbb{E}_{t}^{T_{i}}\left[S\left(T_{i-1}, T_{i}, T_{i+c}\right)\right]$, which we denote by $M_{t}$. By definition, $M_{t}$ is a martingale under $\mathbb{Q}^{T_{i}}$. We conjecture $M_{t}$ is of the form

$$
M_{t}=S\left(t, T_{i}, T_{i+c}\right)+F\left(t, T_{i}, T_{i+c}\right)+G\left(t, T_{i}, T_{i+c}\right) Z_{t}
$$

From (16) we easily obtain the $\mathbb{Q}^{T_{i}}$-dynamics of the swap rate

$$
d S\left(t, T_{i}, T_{i+c}\right) \approx Q_{0}\left(t, T_{i}, T_{i+c}\right) \sigma\left(t, Z_{t}\right) \sigma\left(t, Z_{t}\right)^{*} B\left(t, T_{i}\right) d t+Q_{0}\left(t, T_{i}, T_{i+c}\right) \sigma\left(t, Z_{t}\right) d W_{t}^{T_{i}} .
$$

From (31), we get the $\mathbb{Q}^{T_{i}}$-dynamics of the state variable $Z_{t}$

$$
d Z_{t}=\left[\alpha\left(t, Z_{t}\right)-B\left(t, T_{i}\right) \sigma\left(t, Z_{t}\right)\right] d t+\sigma\left(t, Z_{t}\right) d W_{t}^{T_{i}} .
$$

Then, we can apply Itô to compute dynamics of $M_{t}$. Since $M_{t}$ is a martingale under $\mathbb{Q}^{T_{i}}$, its drift is zero. This condition is a PDE, just like in the LIA case. Taking advantage of the affine structure of the problem, we are able to separate the free terms from the $Z_{t^{-}}$terms and reduce the solution of the PDE to solving the ODE system (39).

\section{Remark 4.10. (Linear Market Model)}

Notice that while the result may seem similar to the linear swap model (LSM) introduced by Hunt and Kennedy (2000), there is a fundamental difference. While the LSM assumes a functional relationship between bond prices and swap rates, our approach implies a relationship between the dynamics of the swaps and the factors underlying the term structure of the interest rates.

\section{References}

Björk, T. (2004). Arbitrage Theory in Continuous Time (2nd ed.). Oxford University Press.

Black, F. (1976). The pricing of commodity contracts. Journal of Financial Economics 3(2), 167-179.

Brace, A. and R.Womersley (2000). Exact fit to the swaption volatility matrix using semidefinite programming. ICBI Global Derivatives Conference, Paris.

Brigo, D. and F. Mercurio (2006). Interest Rate Models: Theory and Practice. Springer Finance, Heidelberg. 2nd edition. 
Cheng, P. and O. Scaillet (2007). Linear-quadratic jump-diffusion modeling. Mathematical Finance 17, 575-598.

Duffie, D., D. Filipović, and W. Schachermayer (2003). Affine processes and applications to finance. The Annals of Applied Probability 13, 984-1053.

Duffie, D., J. Pan, and K. J. Singleton (2000). Transform analysis and asset pricing for affine jump diffusions. Econometrica 68(6), 1343-1376.

Gaspar, R. M. (2004). General quadratic term structures for bond, futures and forward prices. SSE/EFI Working paper Series in Economics and Finance, 559.

Hagan, P. S. (2003). Convexity conundrums: Pricing cms swaps, caps, and floors. Wilmott magazine, 38-44.

Hart, Y. (1997). Unifying theory. RISKe, 54-55.

Hull, J. C. (2006). Options, Futures, and other Derivative Securities. Prentice Hall International, Inc. 6th edition.

Hunt, P. and J. Kennedy (2000). Financial Derivatives in Theory and Practice. John Wiley \& Sons, Chichester.

James, J. and N. Webber (2000). Interest Rate Modelling. John Wiley \& Sons. New York.

Jamshidian, F. (1997). LIBOR and swap market models and measures. Finance and Stochastics 1, 293-330.

Kennedy, D. P. (1997). Characterizing Gaussian models of the term structure of interest rates. Mathematical Finance 7, 107-118.

Pelsser, A. (2000). Efficient Methods for Valuing Interest Rate Derivatives. Springer Finance, Heidelberg.

Plesser, A. (2003). Mathematical foundation of convexity correction. Quantitative Finance 3, 59-65.

Pugachevsky, D. (2001). Forward cms rate adjustment. Risk, 125-128.

Schrager, D. and A. Pelsser (2006). Pricing swaptions and coupon bond options in affine term structure models. Mathematical Finance 16, 673-694. 